\title{
A CHARACTERIZATION OF BIMEASURABLE FUNCTIONS IN TERMS OF UNIVERSALLY MEASURABLE SETS ${ }^{1}$
}

\author{
R. B. DARST
}

Abstract. The purpose of this note is to show, assuming the continuum hypothesis, that a Borel function, $f$, mapping a Borel subset, $D_{f}$, of a separable complete metric space, $M_{1}$, into a separable complete metric space, $M_{2}$, maps Borel subsets of $D_{f}$ onto Borel subsets of $M_{2}$ if, and only if, $f$ maps universally measurable subsets of $D_{f}$ onto universally measurable subsets of $M_{2}$.

Let us begin with some notation and terminology.

Denote by $B_{1}$ and $B_{2}$ the sets of Borel subsets of $M_{1}$ and $M_{2}$.

The statement that a function, $\phi$, is a Borel function from $M_{1}$ to $M_{2}$ means that the domain, $D_{\phi}$, of $\phi$ is an element of $B_{1}$ and $\phi^{-1}\left(B_{2}\right)$ $=\left\{\boldsymbol{\phi}^{-1}(B) ; B \in \mathbb{B}_{2}\right\} \subset \mathbb{B}_{1} \cap D_{\phi}=\left\{B \cap D_{\phi} ; B \in \mathbb{B}_{1}\right\}=\left\{B \in \Re_{1} ; B \subset D_{\phi}\right\}:$ inverse images of Borel sets are Borel sets.

A Borel function, $\phi$, from $M_{1}$ to $M_{2}$ is said to be bimeasurable if $\phi\left(B_{1} \cap D_{\phi}\right) \subset B_{2}$ : images of Borel sets are also Borel sets.

A subset $E$ of a separable metric space, $M$, is said to be universally measurable if the inner measure $\mu_{*}(E)$ is equal to the outer measure $\mu^{*}(E)$ for every probability measure, $\mu$, defined on the Borel subsets of $M$.

Denote by $\mathfrak{u}_{1}$ and $\mathfrak{u}_{2}$ the sets of universally measurable subsets of $M_{1}$ and $M_{2}$.

The main result of this note can now be stated as follows.

TheOREM. Assuming the continuum hypothesis,

$$
f\left(B_{1} \cap D_{f}\right) \subset \otimes_{2} \Leftrightarrow f\left(u_{1} \cap D_{f}\right) \subset u_{2} .
$$

We shall need to employ the continuum hypothesis only in the last step of our argument. If the need to assume it for that step could be circumvented, a much better result would be obtained.

Received by the editors April 18, 1970.

AMS 1969 subject classifications. Primary 0440, 2635, 2810, 2813, 2825, 2865; Secondary 0265, 0274, 2680, 2840.

Key words and phrases. Bimeasurable function, Borel function, Borel set, continuum hypothesis, infinitely differentiable function, probability measure, separable complete metric space, universally measurable function, universally measurable set, universal null set.

1 This research was supported in part by the National Science Foundation under grant GP 9470. 
Before beginning a proof of the theorem, let us recall $[4, \S \S 38-39]$ that Borel functions map Borel sets onto analytic sets and [7, p. 50] analytic sets are universally measurable, so we always have $f\left(B_{1} \cap D_{f}\right) \subset \mathfrak{U}_{2}$. Also, recall that a probability measure, $\mu$, on the Borel subsets of a separable metric space, $M$, has a unique extension to the set of universally measurable subsets of $M$. We shall consider $\mu$ to be so extended. This extension is denoted, without ambiguity, by $\mu$; thus $\mu$ is defined on the analy tic subsets of $M$.

It is well known that if $f$ is an injection, then $f$ maps Borel sets onto Borel sets (e.g. [4, Vol. I, p. 489]). A theorem of Lusin [5, pp. 237-252] shows that if the inverse image, $f^{-1}(y)$, of each point $y$ in $M_{2}$ is a countable subset of $M_{1}$, then $f$ maps Borel sets onto Borel sets. Hence it becomes necessary to look at the set of points $y \in M_{2}$ for which $f^{-1}(y)$ is uncountable, so let

$$
U(f)=\left\{y \in M_{2} ; f^{-1}(y) \text { is uncountable }\right\} .
$$

If $U(f)$ is a countable set, then it is easy to see that $f$ maps Borel sets onto Borel sets. Moreover, if $U(f)$ is countable, then we showed in [2] that $f$ maps universally measurable sets into universally measurable sets. Thus, it remains to consider the case where $U(f)$ is uncountable. If we extend $f$ by making it constant on the Borel set $M_{1}-D_{f}$, we do not change the countability of $U(f)$, so we can assume that $D_{f}=M_{1}$.

Roger Purves showed [6] that if $U(f)$ is uncountable, then $f\left(\Theta_{1}\right)$ $\left\lceil\otimes_{2}\right.$. Purves' paper is the basis for our argument, and we shall of ten refer to it.

At this point, let us recall a bit of recent history. In [1], I showed that if the continuum hypothesis is satisfied, then there exists a real valued continuous function, $\phi$, of bounded variation defined on the interval $I=[0,1]$ such that $\phi$ maps a universally measurable set onto a set which is not Lebesgue measurable. I have recently constructed an infinitely differentiable $\left(C^{\infty}\right)$, real valued function $\psi$ defined on $I$ such that $U(\psi)$ is uncountable. Thus $C^{\infty}$ functions need not map Borel sets onto Borel sets. Moreover, if the continuum hypothesis is assumed, the theorem of this paper implies that $C^{\infty}$ functions need not map universally measurable sets on to universally measurable sets.

Turning now to a proof of our asserted result, assume that $U(f)$ is uncountable. Then $[4$, Vol. I, p. 498] $U(f)$ is an uncountable analytic set.

Purves introduced the notion of similarity of Borel maps $g$ and $h$ and showed that if $g$ and $h$ are similar, then $g$ maps Borel sets onto Borel sets $\Leftrightarrow h$ maps Borel sets onto Borel sets. We shall recall a 
definition of similarity and then establish an analagous proposition for universally measurable sets.

Borel maps $g$ and $h$ from Borel subsets $G$ and $H$ of separable complete metric spaces $M^{1}$ and $M^{2}$ to separable complete metric spaces $M^{3}$ and $M^{4}$ are said to be similar if there exists a one to one Borel map, $\phi$, of $G$ onto $H$ such that $g(x)=g(y) \Leftrightarrow h(\phi(x))=h(\phi(y))$.

(1) If $g$ and h are similar, then

$$
g\left(\mathcal{u}^{1} \cap G\right) \subset \mathcal{u}^{3} \Leftrightarrow h\left(\mathfrak{u}^{2} \cap H\right) \subset \mathcal{u}^{4} .
$$

Proof of (1). Since similarity is easily seen to be an equivalence relation, for our purpose it is sufficient to suppose that $g$ is similar to $h$ and show that the additional supposition $g\left(\mathcal{U}^{1} \cap G\right) \subset \mathcal{u}^{3}$ implies $h\left(\mathcal{u}^{2} \cap H\right) \subset \mathcal{u}^{4}$. To this end, suppose that $E \in \mathcal{u}^{2} \cap H$. Because $\phi$ establishes one to one correspondence between $B^{1} \cap G$ and $B^{2} \cap H$ which extends to a one to one correspondence between $\mathcal{U}^{1} \cap G$ and $\mathcal{u}^{2} \cap H, \phi^{-1}(E) \in \mathcal{U}^{1} \cap G$ which implies that $T=g \circ \phi^{-1}(E) \in \mathcal{U}^{3}$. Define $\psi$ by $\psi(H)=g \circ \phi^{-1} \circ h^{-1}(H)$, so that $\psi$ establishes a one to one correspondence between the sets $a_{g}$ and $a_{h}$ of analytic subsets of the ranges, $h(H)$ and $g(G)$, of $h$ and $g$ and $\psi^{-1}\left(g \circ \phi^{-1}(E)\right)=h(E)$. Let $\lambda$ be the extension to $\mathcal{U}^{4}$ of a probability measure on $B^{4}$, and let $\mu$ be the probability measure defined on $\alpha_{g}$ by $\mu(B)=\lambda\left(\psi^{-1}(B)\right)$. Since $T \in u^{3}$, there are elements $A$ and $B$ of $a_{0}$ such that $A \subset T \subset B$ and $\mu(A)$ $=\mu(B)$. Hence $\psi^{-1}(A) \subset \psi^{-1}(T)=h(E) \subset \psi^{-1}(B)$ and $\lambda\left(\psi^{-1}(A)\right)$ $=\lambda\left(\psi^{-1}(B)\right)$, which imply that $h(E) \in \mathcal{U}^{4}$.

Proposition 5 of [6] tells us that there is a Borel subset $F$ of $M_{1}$ such that the restriction, $f \mid F$, of $f$ to $F$ is similar to a continuous function $g$, defined on the standard Cantor set, $C$, to $M_{2}$, whose range is uncountable and coincides with $U(g)$. We have shown that we can dispense with $f$ and deal with $g$.

Proposition 4 of [6] tells us that there is a Borel subset $G$ of $C$ such that the restriction, $h$, of $g$ to $G$ satisfies

(i) $h^{-1}(y)$ is a perfect subset of $C$ for all $y \in h(G)$,

(ii) $h(G)$ is uncountable.

For our purposes it is necessary to have the following stronger proposition.

(2) There is a closed subset $G$ of $C$ such that the restriction, $h$, of $g$ to $G$ satisfies

(i) $h^{-1}(y)$ is a perfect subset of $C$ for all $y \in h(G)$,

(ii) $h(G)$ is uncountable.

Proof of (2). Denote by $2^{c}$, the compact metric space of closed nonempty subsets of $C$ (cf. [4, Vol. II, $\$ \$ 42-43]$ ). Let $V=g(C)$ and 


$$
S=\left\{(\nu, K) \in V \times 2^{c} ; K \text { is a nonempty perfect subset of } g^{-1}(\nu)\right\} .
$$

Purves showed that $S$ is a Borel set. Since $g(C)=U(g)$ and uncountable analytic sets contain nonempty perfect sets, the projection, $\pi(S)$, of $S$ on its first coordinate is $V$. Purves showed that there is a compact subset $D$ of $S$ such that $\pi(D)$ is uncountable. Then he used a selection theorem from Bourbaki to get his Borel set. We shall construct a Cantor set, $W$, in $D$ such that $\pi \mid W$ is a homeomorphism: Let $\nu_{1}$ and $\nu_{2}$ be distinct condensation points of $\pi(D)$. Let $3 \epsilon_{1}$ be the distance, $\left|\nu_{1}-\nu_{2}\right|$, between $\nu_{1}$ and $\nu_{2}$. Denote the compact, disjoint strips $\left\{(\nu, K) \in D ;\left|\nu-\nu_{i}\right| \leqq \epsilon_{1}\right\}$ by $A_{i}$. Each $A_{i}$ has a finite covering comprised of compact rectangles $A_{i j}=\left\{\nu \in V ;\left|\nu-\nu_{i}\right| \leqq \epsilon_{1}\right\} \times S_{i j}$, where the diameter of each $S_{i j}$ is $\leqq 2 \epsilon_{1}$. Since $\pi\left(A_{i}\right)$ is uncountable, $\pi\left(A_{i j_{i}}\right)$ is uncountable for some $A_{i j_{i}}$ which we denote by $D_{i}$. Iterate this process to obtain a Cantor set $W$ such that $\pi(W)$ is an uncountable compact subset of $V$, and $B_{\nu}=\left\{K \in 2^{c} ;(\nu, K) \in W\right\}$ contains exactly one point, $Q(\nu)$, for all $\nu \in \pi(W)$. The function $\nu \rightarrow Q(\nu)$ is continuous on the compact set $\pi(W)$ and $W$ is its graph. Set $H$ $=\{x \in C ; g(x) \in \pi(W)\}=g^{-1}(\pi(W)) ; H$ is compact since $\pi(W)$ is compact. Set $G=\{x \in H ; x \in Q(g(x))\}$. Recall that $Q(g(x))$ is a nonempty perfect subset of $g^{-1}(g(x))$. If $\nu \notin \pi(W)$, then $G \cap g^{-1}(\nu)=\varnothing$, and if $\nu \in \pi(W)$, then $G \cap g^{-1}(\nu)=Q(\nu)$. It remains to show that $G$ is compact. The map $x \rightarrow Q(g(x))$ is continuous on $H$, so the map $\psi: x$ $\rightarrow(x, Q(g(x)))$, is continuous on $H$. Hence $\psi(H)$ is compact. Also, notice that the set $\Psi=\left\{(x, K) \in C \times 2^{c}, x \in K\right\}$ is closed in $C \times 2^{c}$. Therefore $G=\psi^{-1}(\psi(H) \cap \Psi)$ is compact, and our proof of (2) is completed.

Since $h(G)$ is an uncountable analytic set, $h(G)$ contains a Cantor set, $C_{1}$. Using a similarity map, we can take $C_{1}$ to be $C$. Moreover, $h^{-1}\left(C_{1}\right)$ is a compact subset of $G$ and $h^{-1}\left(C_{1}\right)$ is perfect because $h^{-1}(y)$ is perfect for every $y \in h(G)$. Another similarity then permits us to take $h^{-1}\left(C_{1}\right)$ to be $C$, so we obtain the following proposition which summarizes our progress thus far.

(3) If $U(f)$ is uncountable, then there is a Borel subset $F$ of the domain of $f$ such that the restriction, $f \mid F$, of $f$ to $F$ is similar to a continuous map, $h$, of $C$ onto $C$ satisfying

(i) $h^{-1}(y)$ is a perfect subset of $C$ for all $y \in C$.

Because the domain of $h$ is compact, rather than merely a Borel set, a hard argument of Purves can be extended easily to establish the following proposition.

(4) There exists a Borel map, $s$, of $C$ onto $C$ such that $s \mid h^{-1}(y)$ is a one to one Borel map of $h^{-1}(y)$ onto $C$, for each $y \in C$. 
Purves proves (4) only under the assumption that $h$ is continuous and bimeasurable (i.e., $h(B \cap C) \subset B)$. But, he needs the assumption that $h$ be bimeasurable only at one point in his argument: He needs to assume that $h$ maps relatively compact subsets of its domain onto Borel sets. In our case, the domain of $h$ is compact, so relatively compact subsets of $D_{h}$ are compact and, hence, mapped by $h$ onto compact sets.

Denote $s^{-1}(0)$ by $K$. Then $K$ is an uncountable Borel set. For each $x \in C$, let $r(x)$ be the element of the one element set $h^{-1}(h(x)) \cap K: r(x)$ is the element of $h^{-1}(h(x))$ which is mapped by $s \mid h^{-1}(h(x))$ onto zero. As Purves notes, if $B$ is a Borel set in $C, r^{-1}(B)=\{x \in C ; f(x)=f(y)$ for some $y \in B \cap K\}=f^{-1}(f(B \cap K))$. The latter set is analytic. Likewise $r^{-1}(C-B)$ is analytic, so $r^{-1}(B)$ is Borel. Thus, $r$ is a Borel map of $C$ onto $K$ and the restriction of $r$ to $K$ is the identity. Hence, the map

$$
T: x \rightarrow(r(x), s(x)), \quad x \in C,
$$

is a one to one Borel map of $C$ onto $K \times C$. Moreover, $T$ establishes a similarity between $h$ and the projection map

$$
p:(u, v) \rightarrow u, \quad(u, v) \in K \times C .
$$

Because of (1) our purpose is attained by showing that $p\left(\mathfrak{u}_{a}\right) \nsubseteq \mathfrak{u}_{b}$, where $\mathfrak{U}_{a}$ denotes the universally measurable subsets of $K \times C$ and $\mathfrak{U}_{b}$ denotes the universally measurable subsets of $K$. To this end, let us begin by recalling that a universal null set, $N$, in $K \times C$ is a subset of $K \times C$ satisfying $\mu^{*}(N)=0$ for each nonatomic probability measure, $\mu$, on the Borel subsets of $K \times C$. Remember that subsets of universal null sets are universal null sets and universal null sets are universally measurable. Suppose that there exists a universal null set, $N$, in $K \times C$ satisfying $p(N)=K$. (We have been unable to establish the existence of such a set, $N$, without assuming the continuum hypothesis.) Let $S$ be a subset of $K$ which is not universally measurable and let $E$ $=N \cap p^{-1}(S)$. Then $E \in \mathcal{U}_{a}$ and $p(E)=S \notin \mathcal{U}_{b}$. It remains to assume the continuum hypothesis and establish the existence of $N$. Assume the continuum hypothesis. Let $\left\{\mu_{\alpha}\right\}_{\alpha<\Omega}$ and $\left\{x_{\alpha}\right\}_{\alpha<\Omega}$ be well orderings of the nonatomic probability measures on the Borel subsets of $K \times C$ and the elements of $K$ such that each $\alpha$ has countably many predecessors. For each $\alpha$ there exists a first category $F_{\sigma}$ subset, $F^{\alpha}$, of $C$ such that $\mu_{\alpha}\left(K \times F^{\alpha}\right)=1$ : Look at the probability measure induced on the Borel subsets, $B$, of $C$ by restricting $\mu_{\alpha}$ to sets of the form $K \times B$. Pick $y_{\alpha} \in\left[C-\cup_{\beta \leq \alpha} F^{\beta}\right]$ and let $N=\cup_{\alpha<\Omega}\left(x_{\alpha}, y_{\alpha}\right)$.

Since $N$ intersects each set $K \times F^{\alpha}$ in a countable set and $\mu_{\alpha}$ is nonatomic, $\mu_{\alpha}(N)=0, \alpha<\Omega$. A proof of our theorem is completed 
We conclude with a brief resume:

(a) Purves showed $f\left(\leftrightarrow_{1}\right) \subset \leftrightarrow_{2} \Leftrightarrow U(f)$ is countable.

(b) [2] showed $U(f)$ countable $\Rightarrow f\left(\mathcal{U}_{1}\right) \subset \mathcal{U}_{2}$.

(c) [3] showed $\exists f \in C^{\infty} \ni U(f)$ is uncountable.

(d) (4) showed $U(f)$ uncountable $\Rightarrow \exists F \in B_{1} \ni f \mid F$ is similar to a continuous map, $h$, of $C$ onto $C$ such that $h^{-1}(y)$ is perfect for each $y \in C$.

(e) The Theorem showed $U(f)$ uncountable and the continuum hypothesis $\Rightarrow f(u) \nsubseteq \mathfrak{u}$.

\section{REFERENCES}

1. R. B. Darst, A CBV image of a universal null set need not be a universal null set, Fund. Math. (to appear).

2. - On bimeasurable images of universallym easurable sets, Fund. Math. (to appear).

3. $-C^{\infty}$ functions need not be bimeasurable, Proc. Amer. Math. Soc. 27 (1971), 128-132.

4. K. Kuratowski, Topologie. Vols. 1, 2, Monografie Mat., Toms 20, 21, PWN, Warsaw, 1958, 1961; English transl., Academic Press, New York; PWN, Warsaw, 1966, 1969. MR 19, 873; MR 24 \#A2958.

5. N. Lusin, Leçons sur les ensembles analytiques et leurs applications, GauthierVillars, Paris, 1930.

6. R. Purves, Bimeasurable functions, Fund. Math. 58 (1966), 149-157. MR 33 \#7487.

7. S. Saks, Thérie de l'intégrale, Monografie Mat., Tom 2, PWN, Warsaw, 1933; English transl., Monografie Mat., Tom 7, PWN, Warsaw, 1937.

Purdue University, Lafayette, Indiana 47907 